\section{Learning the laws of lithium-ion electrolyte transport using symbolic regression}

Eibar Flores ${ }^{1}$, Christian Wölke², Peng Yan², Martin Winter ${ }^{2,3}$, Tejs

Vegge $^{1}$, Isidora Cekic-Laskovic ${ }^{2}$ and Arghya Bhowmik ${ }^{1}$

1: Department of Energy Conversion and Storage, Technical

University of Denmark, 2800 Kgs. Lyngby, Denmark.

2: Helmholtz-Institute Münster (IEK-12), Forschungszentrum

Jülich GmbH, Corrensstraße 46, 48149 Münster, Germany.

3: MEET Battery Research Center, University of Münster,

Corrensstrasse 46, 48149 Münster, Germany.

\section{Abstract}

High-throughput experiments (HTE) enable fast exploration of advanced battery electrolytes over vast compositional spaces. Among the multiple properties considered for optimal electrolyte performance, the conductivity is critical. An analytical expression for ionic transport in electrolytes, accurate for practical compositions and operating conditions, would accelerate the process of i) co-optimizing conductivity alongside other desirable electrolyte properties, and ii) learning fundamental physical laws from data, which is one of the paramount goals of scientific big-data analytics. Here, we used symbolic regression with an HTE-acquired dataset of electrolyte conductivity and discovered a simple, accurate, consistent and generalizable surrogate expression. Notably, despite emerging from a purely statistical approach, the expression reflects functional aspects from established thermodynamic limiting laws, indicating our model is grounded on the fundamental physical mechanisms underpinning ionic transport. We prove the potential of using machine learning with HTE to find accurate and physically-sound models in complex systems without established physico-chemical theories.

\section{Introduction}

Non-aqueous aprotic formulations are state-of-the-art electrolytes for Li-ion batteries (LIBs) as they comply with the strict operation requirements for safety, life, reliability and performance. These electrolytes consist of a Li salt dissolved in a mixture of organic solvents, and complemented with performance-enhancing functional additives. Electrolyte formulations balance multiple and often competing properties, among which the ionic conductivity is arguably the most important. ${ }^{1-4}$ The choice of solvents, conducting salts and their proportion usually aims at achieving electrolytes with an optimum mix of low viscosity and high ion dissociation. ${ }^{1,5-7}$ However, the conductivity is not the only electrolyte property to tailor: the electrochemical stability window, chemical compatibility with both electrodes, thermal and chemical stability, liquid range, toxicity and costs, are all important factors to consider. ${ }^{7-11}$ In this multi-objective optimization scenario, researchers in the field would greatly benefit from a predictive, thermodynamic model for electrolyte conductivity, enabling quick exploration of how a promising formulation would affect the electrolytes ionic conductivity without additional experiments. Such a model would ideally be denoted as a simple and universal closed-form expression; i.e., an equation with few algebraic terms, relating easily measurable variables with fundamental physical constants, and without fitting parameters.

Despite significant progress in the thermodynamic description of ionic transport,"12 such a "utopic" model only exists for highly dilute electrolytes. At infinite dilution, the conductivity is simply directly proportional to the ion concentration in solution $c^{13,14}$ However, this model fails at the dilute domain $\left(0<\mathrm{c}<10^{-3} \mathrm{~mol} / \mathrm{L}\right)$ since the conductivity depends additionally on a squared root term of the conducting salt concentration. ${ }^{15}$ Kohlrausch formulated these findings into an empirical law with an adjustable parameter, ${ }^{15,16}$ later addressed by Onsager by considering that ions are dragged not only by hydrodynamic effects, but also by electrophoretic and relaxation phenomena as in the Debye-Hückel theory. The Debye-Hückel-Onsager (DHO) theory effectively upgrades Kohlrausch's law into a fully theoretical law, without adjustable parameters: ${ }^{17}$

$$
\kappa=\kappa^{0}-\left(\frac{z^{2} e F}{3 \pi}\right)\left(\frac{2}{\varepsilon R T}\right)^{1 / 2}\left(\frac{F}{\eta}+\frac{q z \kappa^{0}}{8 \varepsilon R T}\right) c^{3 / 2} \quad \text { Eqn. } 1
$$

where $\kappa^{0}$ is the limiting conductivity, directly proportional to c, and $T, \varepsilon$ and $\eta$ the solution's temperature, permittivity and viscosity, respectively. Despite the success of DHO theory on strong electrolytes, it fails at describing the concentrated ( $c>1$ $\mathrm{mol} / \mathrm{L}$ ) and weak electrolyte formulations used in Li-ion batteries. In its place, researchers formulate expressions following two main approaches. Semi-empirical approaches extend non-electrolyte thermodynamic theories by including long-ranged ion-ion interactions from DHO theory. ${ }^{18,19}$ Instead, phenomenological approaches assume the conductivity to depend on conducting salt concentration and temperature via an arbitrarily-chosen functional expression (e.g. polynomial, exponential), expanded to enough terms to reach a good fit of experimental data; ${ }^{20-22}$ While these models might fit the data well, they are ill-posed to generalize and provide physical insight, given the arbitrary choice of functional expression and all the parameters that need to be adjusted for every new system. Alternatively, a new paradigm of electrolyte engineering employs machine learning run alongside HTE, capable of handling optimization in high dimensional spaces. ${ }^{23,24}$ However, these methods are usually not transferable, hence optimizing for other formulations requires performing new experiments; in addition, little can be learned from a scientific standpoint due to the black-box nature of the underlying process.

In this work we propose an alternative approach -Symbolic Regression (SR) - to find an explainable and accurate model describing the transport of ions in non-aqueous electrolytes. In essence, SR simultaneously learns both adjustable parameters 


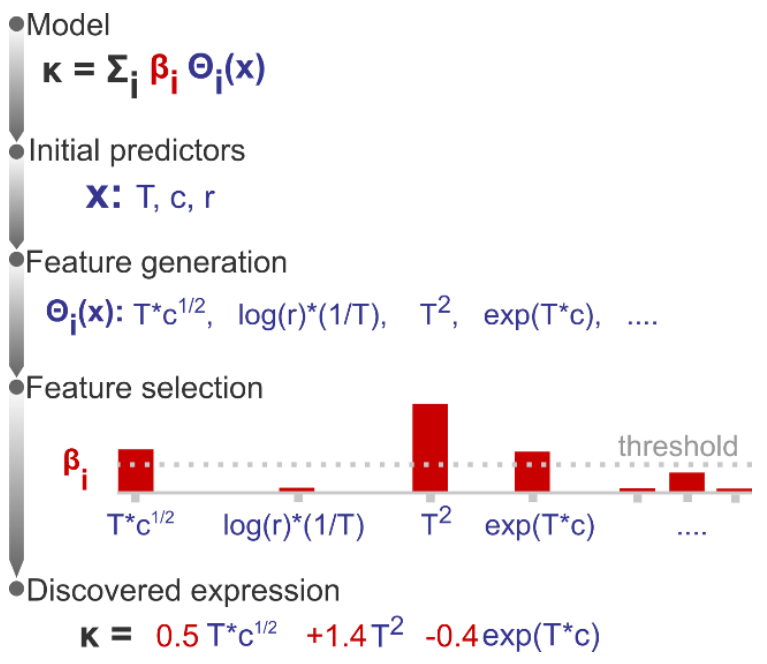

Figure 1. Representation of the symbolic regression method. The conductivity $k$ is represented as a linear model of non-linear transformations of the original predictors: temperature $T$, conducting salt concentration $c$, and PC:EC molar ratio $r$. Based on multiple criteria, only few of the thousands of transformations are selected and used to build an expression.

and the functional form relating electrolyte conductivity with its formulation. We make use of a HTE setup ${ }^{25}$ to collect thousands of conductivity measurements of $\mathrm{LiPF}_{6}$-based electrolytes with ethylene carbonate (EC), propylene carbonate (PC) and ethyl methyl carbonate (EMC) as solvents at different temperatures. For each electrolyte formulation the conductivity was measured in multiple individual samples in order to obtain mean conductivities and standard deviations. The original 3626 individual measurements are thus aggregated into 859 data points. With a simple SR approach, we train multiple candidate expressions. We show that a particular expression emerges as a clear candidate, complying with numerous strict and often competing criteria: i) prediction accuracy, ii) expression simplicity, and iii) model consistency.

In our SR approach, we approximate the conductivity with a generalized linear model, not on the original predictors, but on non-linear transformations of these (i.e. candidate features). Formally:

$$
\kappa \approx \sum_{k} \beta_{k} \boldsymbol{\Theta}_{k}(T, c, r) \quad \text { Eqn. } 2
$$

where $\beta_{k}$ are the $\mathrm{k}^{- \text {th }}$ coefficient and $\boldsymbol{\Theta}_{\boldsymbol{k}}$ the $\mathrm{k}^{\text {th }}$ transformation on the predictors: temperature $T$, conducting salt concentration $c$ and PC:EC molar ratio $r$. The conductivity is assumed to depend not on all possible candidate features, but on a much-reduced set of these; i.e., the solution of Eqn. 2 is sparse. Figure 1 illustrates the methodology, split into feature generation and selection steps. Briefly, the training process involves defining a transformation set (e.g. inverse, logarithms, exponentials), then applying it to the initial predictors to generate a library of

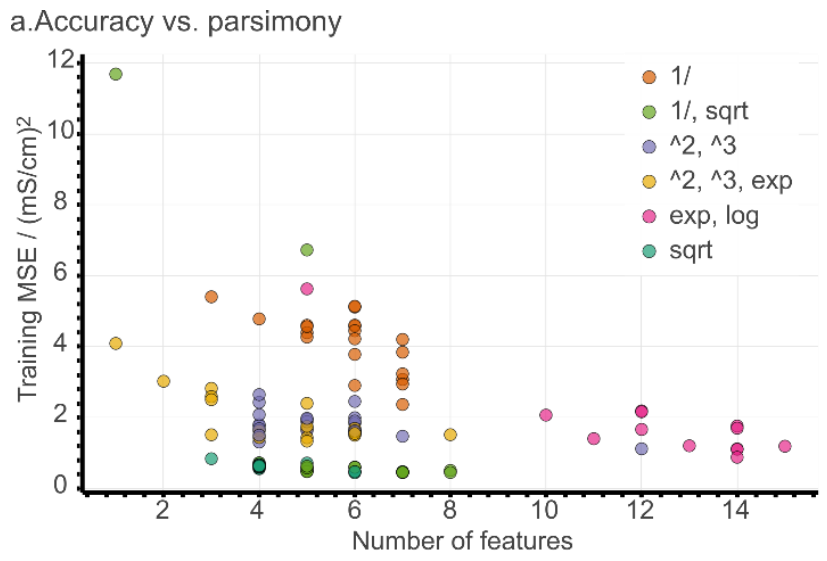

b. Consistency

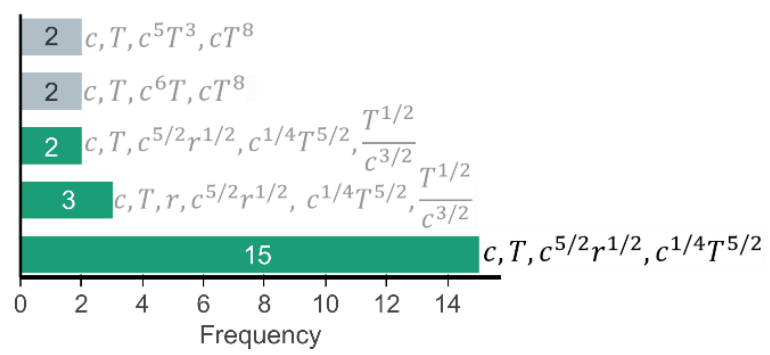

Figure 2. Accuracy, parsimony and consistency of discovered expressions throughout multiple training sessions. All expressions were trained with the constrain $k_{0}=0$.

candidate features, a few of which are selected to form a candidate expression.

The discovered expressions are not unique: candidate features might combine in multiple ways to result in similarly accurate expressions. Consequently, instead of using all 515 samples on the training set, we train instead on multiple, random subsamples of 50,100, 250 and 400 data points in order to evaluate whether a discovered expression is consistent across a total of 20 independent training sessions. We use the validation set to evaluate the performance of the discovered expressions and compare them to four benchmark models (Supplementary Information, Table 4). The first benchmark uses the three initial predictors as the simplest approximation. The second uses $3^{\text {rd }}$ order polynomial expansions of the initial predictors, as in phenomenological models. ${ }^{26}$ The third assumes exponential transformations as in Arrhenius-based models. The fourth uses exponential transformations on the $3^{\text {rd }}$-order polynomial expansion of the initial predictors, such as in the extended Castel-Amis model. ${ }^{6}$

During the evaluation, we search for an expression being not only i) accurate, i.e., yielding a low mean squared error (MSE), but also ii) parsimonious, quantified as the number of terms of the expression, and iii) stable, represented by the number of times the expression repeats across training sessions. Figure $2 \mathrm{a}$ presents the accuracy vs. complexity trade-off from the expressions found. Each data point represents an expression, 
whose color references its parent transformation set. As expected, larger expressions fit the data better, however, at the expense of increased complexity; this is the case of the expressions originating from exponential and logarithmic transformations (MSE $<2$ but $10+$ terms). Interestingly, the expressions populating the Pareto-frontier of the figure originate from transformation sets including square-root operations; i.e., they offer the best compromise between MSE and the number of terms.

Note that most expressions only appear once, highlighting these to be highly sensitive to the training subsample and that there is no unique solution. Figure $2 b$ shows the most frequent expressions across the training sessions, where expressions with square-root operations are highlighted in green. Unlike most expressions, the model:

$$
\kappa=\beta_{1} c+\beta_{2} T+\beta_{3} c^{5 / 2} r^{1 / 2}+\beta_{4} c^{1 / 4} T^{5 / 2}
$$

Eqn. 3

is by far the most frequent and was discovered 15 times out of 20 training sessions. We, therefore, select this expression as it clearly stands out from the other competing models, for being not only consistent but also parsimonious (four terms), comparatively accurate in the training set (MSE $<0.75$ ), and generalizable, as evidenced by a good accuracy in the validation set. Table 1 summarizes the coefficients and performance metrics of the selected expression Eqn. 3 .

Table 1. Coefficients of Eqn. 3 and associated performance metrics after training on the full training set of 515 samples.

\begin{tabular}{|c|c|}
\hline Attribute & Value \\
\hline$\beta_{1}$ & --5.11 \\
\hline$\beta_{2}$ & --0.040 \\
\hline$\beta_{3}$ & -0.35 \\
\hline$\beta_{4}$ & $2.73 \times 10^{-4}$ \\
\hline Training MSE & 1.08 \\
\hline Training $\mathrm{R}^{2}$ & 0.92 \\
\hline Validation MSE & 1.22 \\
\hline Validation $\mathrm{R}^{2}$ & 0.90 \\
\hline
\end{tabular}

Constraining models might be beneficial when selecting promising surrogate models. To illustrate why, we repeated the 20 training sessions with the same transformation sets, this time allowing the intercept to vary freely. The corresponding training errors and stability histograms are shown in Supplementary Figures 4 and 5. Expectedly, removing the intercept constrain results in slightly improved accuracy but significantly aggravates model stability. Implementing domain-knowledge constraints is clearly an effective filter to discover consistent and physicallysound expressions. However, constrained models have significantly higher data requirements. Figure S6 shows the learning curves of the discovered expression, retrained on subsamples of different sizes with and without the intercept constraint. The non-constrained expression converges to a. Validation rMSE and measurement dispersion

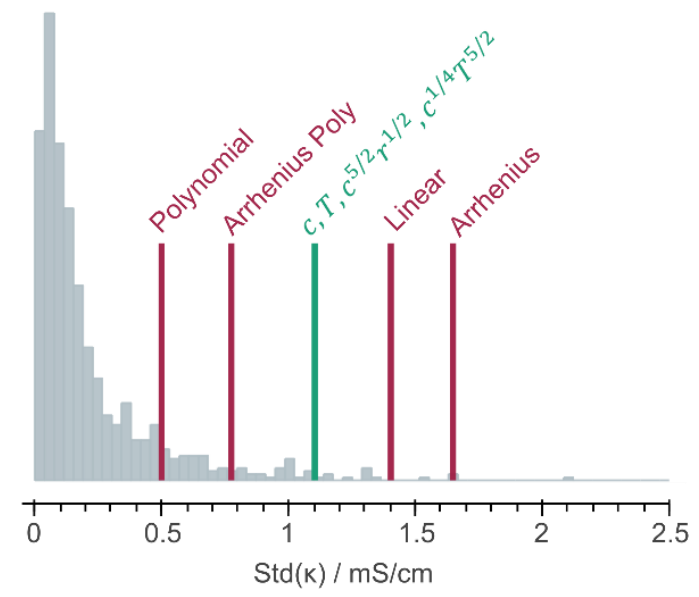

b. Fit on withheld set

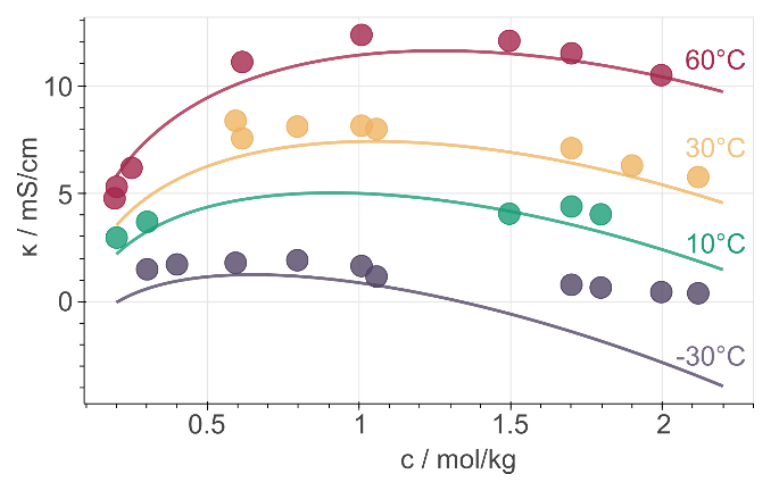

Figure 3: a. Root mean square error of selected model (green) and benchmarks (red) on the validation set, compared to measurement dispersion (grey). $b$. Fit of the selected model on the withheld (validation and test) set at $r=1.0$.

optimal accuracy already with 100 samples; in contrast, the constrained model fails at almost all samples sizes and only approaches optimal accuracy when using all 515 training samples. Such data requirements need to be weighed when modelling the often-small datasets available from experiments.

Figure 3a compares the accuracy of the selected constrained expression on the validation set, relative to the measurement dispersion and along with benchmark models. We use the root mean squared error ( $\mathrm{rMSE}$ ) to describe the prediction accuracy in the same units $[\mathrm{mS} / \mathrm{cm}]$ as the conductivity measurements. As expected, the simpler benchmarks such as Linear and Simple Arrhenius models are less accurate. Instead, the more complex models (Polynomial and Arrhenius Polynomial) are prone to overfitting, as their prediction errors are smaller than a nonnegligible fraction of measurement dispersion values. Notably, the selected model stands in the middle with a validation-set rMSE of $1.1 \mathrm{mS} / \mathrm{cm}$, indicating that it is accurate up to the measurement noise and so it does not overfit the dataset. At first glance, the selected model seems to yield only a minor improvement $(0.3 \mathrm{mS} / \mathrm{cm})$ compared to the basic linear model; 
however, the square-root dependence in the selected model reproduces the curvature and maxima in the data and ii) by having no intercept, it complies with the physical constraint of no conductivity at $\mathrm{c}, \mathrm{T}, \mathrm{r}=0$.

Figure $3 b$ illustrates that the selected model generally fits well the data not used in the training (i.e., validation and testing sets). However, the fit generally underestimates the measurements. The same expression trained with an intercept (Figure S7) fits the withheld data without such bias, indicating that the underestimation in Figure $3 b$ is a result of imposing the physically-motivated $\mathrm{y}_{0}=0$ constraint. In addition, the model is not flexible enough to describe the conductivities measured at $-30{ }^{\circ} \mathrm{C}$ and concentrations $>1 \mathrm{~mol} / \mathrm{kg}$; in these extreme regimes the high salt concentration and low temperature result in a highly viscous medium that might promote a non-vehicular type of ionic transport. ${ }^{27}$

However, we highlight that in most of the experimental range the fit reproduces the concentration- and temperaturedependent conductivity maxima observed in the data and in previous studies, which is a key attribute for implementing the discovered model as part of multi-target optimization and/or active learning frameworks. ${ }^{24}$

Assigning a physical meaning to the discovered expression is not straightforward. For one, any comparison to the thermodynamically-derived DHO law would require to explicitly account for the solution's viscosity and dielectric constant, measurements that are not available in the dataset. Second, there are no constraints to avoid unphysical values, like the negative conductivities at sub-zero temperatures and high conducting salt concentrations (Figure $3 \mathrm{~b}$ ). Third, the solution to our symbolic regression approach is generally not unique, i.e., there are multiple expressions equally accurate to fit the dataset. Despite these limitations, we observe that expressions sharing square-root transformations achieve the best compromise between simplicity and accuracy. Therefore, we believe that our method is learning square-root trends inherent to the data manifold, which indicates that some functional aspects of the DHO law - i.e. its square-root trends on temperature and concentration - are still valid to describe electrolyte conductivity in concentrated formulations.

Physical insights can be drawn not only from the expression itself but also from its predictions. Figure 4 illustrates the conductivity trends from our selected model within the space of electrolyte formulations used for the training. As expected, at higher temperatures, the conductivity increases and the conductivity maxima shift towards higher salt concentrations $(0.74 \mathrm{~mol} / \mathrm{kg}$ at $-30{ }^{\circ} \mathrm{C}$ to $1.70 \mathrm{~mol} / \mathrm{kg}$ at $60{ }^{\circ} \mathrm{C}$, see Fig. $3 \mathrm{~b}$ ). However, the role of the cyclic carbonate is subtler. Note first that all conductivities peak when the electrolyte formulation is EC-pure (PC:EC ratio = 0 ). Second, the tails along the salt concentration axis elongate at higher concentrations as the formulations become increasingly EC-pure. From a fundamental standpoint, conductivity depends a. Conductivity at $-30^{\circ} \mathrm{C}$

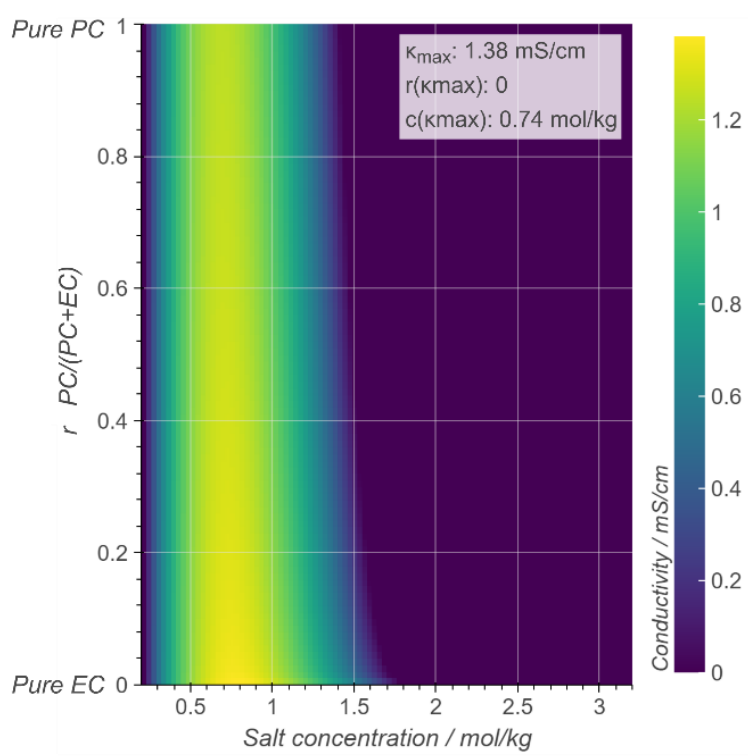

b. Conductivity at $60^{\circ} \mathrm{C}$

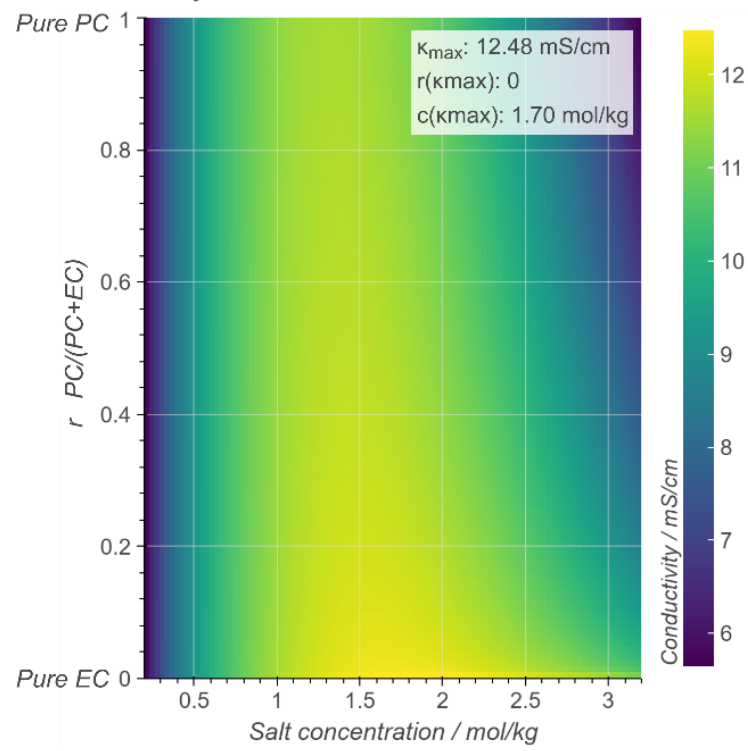

Figure 4. Contour maps of electrolyte conductivity versus PC:EC ratio and conducting salt concentration, as predicted by our selected and trained model (Eqn.3) at a. low and b. high operating temperatures.

on a compromise between the ionic mobility, mainly influenced by viscosity, and the number of charge carriers available for migration, mainly controlled by the electrolyte's dielectric constant (c.f. see Bjerrums criterion ${ }^{1}$ for ionic association). ${ }^{5,6}$ As EC has a higher dielectric constant compared to PC, ${ }^{28}$ EC-pure solutions are more effective at preventing ion association and so enhance electrolyte conductivity. This effect is especially pronounced at high conducting salt concentrations, as indicated in the tails in Figure 4, where ionic association becomes a critical limiting factor for ion transport in the electrolyte. ${ }^{5,29}$ Such EC- 
driven improvement of conductivity, which has been observed experimentally, ${ }^{30}$ is not evident neither in the correlation maps in Supplementary Figure 2 nor the pair-plots Supplementary Figure 3. Despite this, our SR approach manages to capture these subtle effects that align with our current understanding of the interplay between the solvent's dielectric properties and ionic migration.

At this point, we emphasize we have only implemented two domain-knowledge decisions - include square-root transformations and constrain the intercept to zero - on an otherwise purely statistical approach. Even in these circumstances, we observe the emergence of an expression clearly outstanding from competing models, for being accurate without overfitting, simple, consistent, with a square-root functional structure resembling the DHO law, and agreeing with our understanding of ionic migration. In other words, our expression is not only an appropriate surrogate model from a machine-learning standpoint but also seems grounded on the physical-chemical mechanisms underpinning ion transport in electrolytes. Our work opens multiple avenues to pursue further the data-driven discovery of accurate surrogate models capable of bridging the existing gap ${ }^{19}$ in the understanding of concentrated electrolyte formulations. To start with, atomistic descriptors can be incorporated in order to generalize to solvent mixtures other than PC/EC/EMC and conducting salt chemistries beyond conventional Li-ion technology. ${ }^{21}$ In addition, using other promising SR algorithms ${ }^{31}$ and implementing domain-knowledge constraints in the feature selection step ${ }^{32}$ could alleviate the issue with expression consistency and yield physically-sound expressions; i.e. rigorous to known boundary conditions (e.g. $\kappa(c=0)=0$ ) and to asymptotic behavior on key limits (e.g. $\left.\lim _{c \rightarrow 0} \kappa \propto c\right)$. These constraints will have to be carefully balanced, given our observations of the data-intensive nature of constrained models.

In summary, we apply symbolic regression as a data-driven method to learn the effects of temperature, conducting salt concentration and solvent ratio on the conductivity of a concentrated electrolyte. We use a dataset of 859 experimental measurements on a $\mathrm{LiPF}_{6}$ in EC, PC and EMC electrolyte at different temperatures, conducting salt concentrations and EC-to-PC ratios within the practical ranges of operation of Libased battery electrolytes. Our approach generates thousands of derived features from the initial predictors using a set of nonlinear transformations. Few of the derived features are then selected using cross-validated Lasso regression to discover candidate expressions, which are then compared in terms of accuracy, simplicity, and consistency. We find that expressions in the Pareto-frontier share a square-root form, which we believe reflects an underlying data manifold resembling the Debye-Hückel-Onsager equation. Out of these expressions, we singled out a 4-term expression for being consistent, accurate and simple. The discovered expression does not overfit the data fits the withheld set well, and reproduces the conductivity behavior expected from similar theoretical and experimental studies. The discovered expression is a promising surrogate model to be used in multi-variable electrolyte optimization. More broadly, the presented methodology can be used to find surrogate expressions to physicochemical systems where no closed-form solution exists. Implementing phenomenological constraints in the feature selection step, keeping in mind the inherently high data requirements, would significantly support the search for physically-sound surrogate expressions using symbolic regression.

\section{Associated content}

Supporting Information. Experimental details, exploratory data analysis, hyperparameters for feature generation and selection, accuracy, simplicity, consistency and fit on the withheld set from unconstrained models, learning curves.

Code availability. The code to train symbolic regression models, along with examples, are openly available in the Github repository: https://github.com/BIG-MAP/SR-electrolytes.

Data availability. The dataset is openly available as a supplementary file. The predictions from the trained model can be further explored in the following web site: https://bigmap.github.io/SR-electrolytes/.

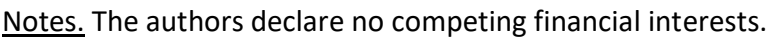

\section{Acknowledgments}

This project has received funding from the European Union's Horizon 2020 research and innovation initiative under grants agreement No 957189 (BIG-MAP) and No 957213 (BATTERY 2030+). EF acknowledges Prof. Patrik Johansson for fruitful discussions.

\section{References}

(1) Xu, K. Nonaqueous Liquid Electrolytes for LithiumBased Rechargeable Batteries. Chem. Rev. 2004, 104 (10), 4303-4417.

(2) Xu, K. Electrolytes and Interphases in Li-lon Batteries and Beyond. Chem. Rev. 2014, 114 (23), 11503-11618.

(3) Schmitz, R. W.; Murmann, P.; Schmitz, R.; Müller, R.; Krämer, L.; Kasnatscheew, J.; Isken, P.; Niehoff, P.; Nowak, S.; Röschenthaler, G. V.; Ignatiev, N.; Sartori, P.; Passerini, S.; Kunze, M.; Lex-Balducci, A.; Schreiner, C.; Cekic-Laskovic, I.; Winter, M. Investigations on Novel Electrolytes, Solvents and SEI Additives for Use in Lithium-Ion Batteries: Systematic Electrochemical Characterization and Detailed Analysis by Spectroscopic Methods. Prog. Solid State Chem. 2014, $42(4), 65-84$.

(4) Schmuch, R.; Wagner, R.; Hörpel, G.; Placke, T.; Winter, $M$. Performance and Cost of Materials for Lithium- 
Based Rechargeable Automotive Batteries. Nat. Energy 2018, 3 (4), 267-278.

(5) Seo, D. M.; Borodin, O.; Balogh, D.; O'Connell, M.; Ly, Q.; Han, S.-D.; Passerini, S.; Henderson, W. A. Electrolyte Solvation and Ionic Association III. Acetonitrile-Lithium Salt Mixtures-Transport Properties. J. Electrochem. Soc. 2013, 160 (8), A1061A1070.

Ding, M. S.; Jow, T. R. Physicochemical Properties of Non-Aqueous Solvents and Electrolytes for Lithium Battery Applications. ECS Trans. 2009, 16 (35), 183214.

(7) Cekic-Laskovic, I.; von Aspern, N.; Imholt, L.; Kaymaksiz, S.; Oldiges, K.; Rad, B. R.; Winter, M. Synergistic Effect of Blended Components in Nonaqueous Electrolytes for Lithium Ion Batteries. Top. Curr. Chem. 2017, 375 (2), 1-64.

(8) Armand, M.; Axmann, P.; Bresser, D.; Copley, M.; Edström, K.; Ekberg, C.; Guyomard, D.; Lestriez, B.; Novák, P.; Petranikova, M.; Porcher, W.; Trabesinger, S.; Wohlfahrt-Mehrens, M.; Zhang, H. Lithium-Ion Batteries - Current State of the Art and Anticipated Developments. J. Power Sources 2020, 479, 228708.

(9) Xu, K.; Von Cresce, A. Interfacing Electrolytes with Electrodes in Li Ion Batteries. J. Mater. Chem. 2011, 21 (27), 9849-9864.

(10) Nowak, S.; Winter, M. Review-Chemical Analysis for a Better Understanding of Aging and Degradation Mechanisms of Non-Aqueous Electrolytes for Lithium Ion Batteries: Method Development, Application and Lessons Learned. J. Electrochem. Soc. 2015, 162 (14), A2500-A2508.

(11) von Aspern, N.; Röschenthaler, G. V.; Winter, M.; Cekic-Laskovic, I. Fluorine and Lithium: Ideal Partners for High-Performance Rechargeable Battery Electrolytes. Angew. Chemie - Int. Ed. 2019, 58 (45), 15978-16000.

(12) Dufrêche, J.-F.; Bernard, O.; Durand-Vidal, S.; Turq, P. Analytical Theories of Transport in Concentrated Electrolyte Solutions from the MSA. 2005.

(13) Atkins, P.; Paula, J. de. Molecules in Motion. In Phyiscal Chemistry; Oxford University Press: Oxford, 2006; pp 747-790.

(14) Matsuda, Y.; Morita, M.; Kosaka, K. Conductivity of the Mixed Organic Electrolyte Containing Propylene Carbonate and 1,2-Dimethoxyethane. J. Electrochem. Soc. 1983, 130 (1), 101-104.

(15) Martínez, L. MEASURING THE CONDUCTIVITY OF VERY DILUTE ELECTROLYTE SOLUTIONS, DROP BY DROP Quim. Nova 2018, 23 (7), 341.
(16) Duncan A., M. I. The Conductance of Aqueous Solutions of Electrolytes. J. Franklin Inst. 1938, 225 (6), 661-686.

Onsager, L. Report on a Revision of the Conductivity Theory. Trans. Faraday Soc. 1927, 23, 341.

Anderko, A.; Wang, P.; Rafal, M. Electrolyte Solutions: From Thermodynamic and Transport Property Models to the Simulation of Industrial Processes. Fluid Phase Equilib. 2002, 194-197, 123-142.

(19) Kontogeorgis, G. M.; Maribo-Mogensen, B.; Thomsen, $K$. The Debye-Hückel Theory and Its Importance in Modeling Electrolyte Solutions. Fluid Phase Equilib. 2018, 462, 130-152.

Gilliam, R. J.; Graydon, J. W.; Kirk, D. W.; Thorpe, S. J. A Review of Specific Conductivities of Potassium Hydroxide Solutions for Various Concentrations and Temperatures. Int. J. Hydrogen Energy 2007, 32 (3), 359-364.

Nilsson-Hallén, J.; Ahlström, B.; Marczewski, M.; Johansson, P. Ionic Liquids: A Simple Model to Predict Ion Conductivity Based on DFT Derived Physical Parameters. Front. Chem. 2019, 7 (MAR), 1-6.

Landesfeind, J.; Gasteiger, H. A. Temperature and Concentration Dependence of the Ionic Transport Properties of Lithium-Ion Battery Electrolytes. J. Electrochem. Soc. 2019, 166 (14), A3079-A3097.

(23) Matsuda, S.; Nishioka, K.; Nakanishi, S. HighThroughput Combinatorial Screening of MultiComponent Electrolyte Additives to Improve the Performance of Li Metal Secondary Batteries. Sci. Reports 201991 2019, 9 (1), 1-8.

Dave, A.; Mitchell, J.; Kandasamy, K.; Wang, H.; Burke, S.; Paria, B.; Póczos, B.; Whitacre, J.; Viswanathan, V. Autonomous Discovery of Battery Electrolytes with Robotic Experimentation and Machine Learning. Cell Reports Phys. Sci. 2020, 1 (12).

Krishnamoorthy, A. N.; Wölke, C.; Diddens, D.; Maiti, M. Data-Driven Analysis of High-Throughput Experiments on Liquid Battery Electrolyte Formulations : Unraveling the Impact of Composition on Conductivity. ChemRxiv Prepr. 2022, 1-22.

Valøen, L. O.; Reimers, J. N. Transport Properties of LiPF6-Based Li-Ion Battery Electrolytes. J. Electrochem. Soc. 2005, 152 (5), A882.

Flores, E.; Åvall, G.; Jeschke, S.; Johansson, P. Solvation Structure in Dilute to Highly Concentrated Electrolytes for Lithium-Ion and Sodium-Ion Batteries. Electrochim. Acta 2017, 233, 134-141.
Hall, D. S.; Self, J.; Dahn, J. R. Dielectric Constants for Quantum Chemistry and Li-Ion Batteries: Solvent 
Blends of Ethylene Carbonate and Ethyl Methyl

Carbonate. J. Phys. Chem. C 2015, 119 (39), 22322-

22330.

(29) Seo, D. M.; Borodin, O.; Han, S.-D.; Boyle, P. D.; Henderson, W. A. Electrolyte Solvation and Ionic Association II. Acetonitrile-Lithium Salt Mixtures: Highly Dissociated Salts. J. Electrochem. Soc. 2012, 159 (9), A1489-A1500.

(30) Ding, M. S.; Jow, T. R. Conductivity and Viscosity of PCDEC and PC-EC Solutions of LiPF[Sub 6]. J. Electrochem. Soc. 2003, 150 (5), A620.

(31) Broløs, K. R.; Machado, M. V.; Cave, C.; Kasak, J.; Stentoft-Hansen, V.; Batanero, V. G.; Jelen, T.; Wilstrup, C. An Approach to Symbolic Regression Using Feyn. 2021.

(32) Cornelio, C.; Dash, S.; Austel, V.; Josephson, T.; Goncalves, J.; Clarkson, K.; Megiddo, N.; Khadir, B. El; Horesh, L. Al Descartes: Combining Data and Theory for Derivable Scientific Discovery. 2021, 1-26. 